\title{
Fatores que difundem a assistência de enfermagem humanizada na unidade de terapia intensiva
}

\author{
Factors that spread in humanized nursing care in the intensive care unit
}

Factores que se propagan en los cuidados de enfermería humanizados en una unidad de cuidados intensivos

Italo Everton Bezerra Barbosa ${ }^{1 *}$, Breno de Souza Mota ${ }^{2}$, Alicia Ribeiro Fonseca ${ }^{1}$, Daniella Souza Gama Siqueira ${ }^{3}$, Fabrício de Souza Melo ${ }^{3}$, Felipe Chrystian de Figueiredo Lira ${ }^{1}$, Kalinny Izel de Souza Marques ${ }^{4}$, Misaele Silva Maciel ${ }^{1}$, Nataly Danielle Araújo Queiroz ${ }^{5}$, Theodora Maria de Paiva dos Santos ${ }^{6}$.

\section{RESUMO}

Objetivo: Compreender fatores que estejam envolvidos na promoção e integralidade da assistência de enfermagem prestada ao paciente em uma UTI, atentando para a percepção da equipe de enfermagem sobre a importância da assistência humanizada e os possíveis fatores que interferem nesse processo. Métodos: Trata-se de uma pesquisa descritiva, utilizando como técnica a Revisão Integrativa da Literatura (RIL). O levantamento de dados foi realizado entre os meses de dezembro de 2020 e fevereiro de 2021, nas bases de dados da Biblioteca Virtual de Saúde (BVS) e Scientific Electronic Library Online (SCIELO), utilizando os seguintes descritores em saúde: Enfermagem AND Humanização AND Unidade de Terapia Intensiva. Foram encontradas 280 publicações na totalidade, após a aplicação dos critérios de elegibilidade e inelegibilidade, 15 publicações foram selecionadas para compor essa pesquisa. Resultados: Os fatores que contribuem para assistência humanizada são: boa comunicação interpessoal, infraestrutura da área hospitalar e capacitação dos profissionais. Referente aos fatores que dificultam a assistência humanizada: falta de conhecimento e a diminuição do quadro de funcionários. Considerações Finais: Diante disso, observou-se que os profissionais de enfermagem compreendem como funciona o processo de humanização, porém alguns fatores interferem nesse processo. Logo, torna-se extremamente válido que as instituições realizem capacitações periódicas nesses profissionais.

Palavras-chave: Humanização da assistência, Profissionais de enfermagem, Unidades de terapia intensiva.

\begin{abstract}
Objective: To understand factors that are involved in the promotion and comprehensiveness of nursing care provided to the patient in an ICU, paying attention to the perception of the nursing team about the importance of humanized care and the possible factors that interfere in this process. Methods: This is a descriptive research, using the Integrative Literature Review (RIL) as a technique. The data survey was carried out between the months of December 2020 and February 2021, in the databases of the Virtual Health Library (VHL) and Scientific Electronic Library Online (SCIELO), using the following health descriptors: Enfermagem

1 Centro Universitário FAMETRO (CEUNI-FAMETRO), Manaus - AM. *E-mail: italoeverton1998@gmail.com

2 Universidade de São Paulo (USP), São Paulo - SP.

${ }^{3}$ Instituto Singular Educacional, Manaus - AM.

${ }^{4}$ Faculdade Estácio Amazonas, Manaus - AM.

${ }^{5}$ Líder Instituto Educacional, Manaus - AM.

${ }_{6}^{6}$ Universidade Federal de Minas Gerais, Belo Horizonte - MG.
\end{abstract}


AND Humanização AND Intensive care unit. A total of 280 publications were found, after applying the eligibility and ineligibility criteria, 15 publications were selected to compose this research. Results: The factors that contribute to humanized assistance are good interpersonal communication, infrastructure in the hospital area and training of professionals. Regarding the factors that hinder humanized assistance: lack of knowledge and the decrease in the number of employees. Final Considerations: In view of this, it was observed that nursing professionals understand how the humanization process works, however, some factors interfere in this process. Therefore, it is extremely valid that institutions carry out periodic training on these professionals.

Key words: Humanization of assistance, Nurse practitioners, Intensive care units.

\section{RESUMEN}

Objetivo: Comprender los factores que intervienen en la promoción e integralidad del cuidado de enfermería brindado al paciente en una $\mathrm{UCl}$, prestando atención a la percepción del equipo de enfermería sobre la importancia del cuidado humanizado y los posibles factores que interfieren en este proceso. Métodos: Se trata de una investigación descriptiva, utilizando como técnica la Revisión Integrativa de Literatura (RIL). O levantamento de dados foi realizado entre os meses de dezembro de 2020 e fevereiro de 2021, nas bases de dados da Biblioteca Virtual de Saúde (BVS) e Scientific Electronic Library Online (SCIELO), utilizando os seguintes descritores em saúde: Enfermagem AND Humanização AND Unidad de terapia intensiva. Se encontraron un total de 280 publicaciones, luego de aplicar los criterios de elegibilidad e inelegibilidad, se seleccionaron 15 publicaciones para componer esta investigación. Resultados: Los factores que contribuyen a la asistencia humanizada son: buena comunicación interpersonal, infraestructura en el área hospitalaria y formación de profesionales. Respecto a los factores que dificultan la asistencia humanizada: desconocimiento y disminución del número de empleados. Consideraciones finales: Ante esto, se observó que los profesionales de enfermería comprenden cómo funciona el proceso de humanización, sin embargo, algunos factores interfieren en este proceso. Por tanto, es sumamente válido que las instituciones realicen formaciones periódicas a estos profesionales.

Palabras clave: Humanización de la atención, Enfermeras practicantes, Unidades de cuidados intensivos.

\section{INTRODUÇÃO}

A Unidade de Terapia Intensiva (UTI) é uma área hospitalar destinada ao atendimento de pacientes que se encontram em estado crítico, com chances de sobrevida, que necessitam de cuidados e monitoramento intensivos, através de mecanismos e tecnologias avançadas, em conjunto com profissionais especializados, tendo como foco não somente o tratamento da patologia, mas também para questões ambientais, psicossociais e familiares (BARBOSA IEB, et al., 2021).

A UTI é um ambiente incerto e desconhecido, que traz aos familiares e pacientes, uma ideia de gravidade, associada à morte, que muitas vezes não se condiz com a realidade. A internação na UTI é um dos principais momentos que desencadeiam possíveis crises de estresse, não somente na equipe de enfermagem, mas também nos familiares e principalmente nos pacientes (SANTOS ES, et al., 2016).

Nessa perspectiva, a UTI é um ambiente hospitalar onde existe uma maior chance de desenvolver distúrbios psicológicos e emocionais nos pacientes, uma vez que existe uma maior possibilidade de estes presenciarem emergências e de morte, tornando assim o convívio mais tenso e hostil. Com isso, é de extrema importância que a equipe multiprofissional esteja preparada e capacitada para agir mediante a qualquer intercorrência, prestando uma assistência segura e humanizada (MACHADO ER, et al., 2016).

Ao longo do tempo, aliado aos recursos tecnológicos, o serviço prestado pelo profissional ao paciente e familiares acabou se robotizando ou se tornando menos direto. Isto repercute diretamente na qualidade da assistência prestada e na automatização do cuidado. O enfermeiro está inserido como principal gestor nessa situação, além de ser o responsável pela equipe de enfermagem, está diretamente envolvido com o atendimento prestado ao paciente (LUIZ FF, et al., 2017). 
Nesse contexto, prestar uma assistência segura e humanizada configura-se como algo de extrema relevância para recuperação dos pacientes. A humanização em uma UTI significa ter uma visão holística durante toda a assistência prestada, envolvendo um conjunto de iniciativas que visem a produção de cuidados com respeito cultural e ético, aumentando a eficácia e adesão do tratamento, em conjunto com a satisfação dos pacientes, para que seja possível estreitar as relações em ambas as partes (MASSAROLI R, et al., 2015).

Em relação à humanização no Brasil, existem problemas no cotidiano que dificultam a efetivação dessa ação tanto nas redes públicas e/ou privadas, dentre eles o tratamento focado somente nas complicações, esquecendo o paciente como pessoa humana. Surge então, uma necessidade de criar modelos assistenciais e estratégias, visando $o$ atendimento seguro, respeitando assim os valores humanos e ideais dos pacientes (SILVA JC, et al., 2016).

$\mathrm{Na}$ atualidade, onde o conhecimento está diretamente ligado como principal fator de produção, tratar o paciente de maneira humanizada torna-se uma atividade essencial dentro de uma área hospitalar. Cabe ressaltar que algumas condições do trabalho podem acabar interferindo na qualidade da assistência prestada, como a falta de medicamentos e insumos para procedimentos, como seringas, agulhas, luvas, ou até mesmo produtos para lavagem das mãos, que é algo fundamental no controle de infecções (MALTA DC, et al., 2018).

À medida que novas tecnologias vão sendo implementadas dentro da UTI, torna-se necessária uma qualificação dos profissionais sobre atendimentos humanizados, com segurança, precisão e eficácia, preservando os valores éticos e humanísticos dos pacientes. As iniciativas de uma assistência de enfermagem humanizada estão diretamente ligadas com a qualidade do serviço de saúde prestado ao paciente, configurando-se como um grande desafio para equipe de enfermagem (PASCUCI L, et al., 2017).

Diante disso, o Programa Nacional de Humanização da Assistência Hospitalar (PNHAH), discute a qualidade da assistência que é prestada aos pacientes durante seu atendimento, pontuando que essa assistência é um grande fator de preocupação para o sistema de saúde no Brasil. Através disso, foram traçadas medidas que buscam difundir uma nova cultura voltada para humanização na rede hospitalar, com o objetivo de capacitar os profissionais, introduzindo um novo conceito sobre assistência humanizada, valorizando a cidadania e a vida humana, além de fortalecer as estratégias articuladas já existentes no sistema de saúde (SANTOS ES, et al., 2016).

Nesse contexto, o programa de Educação Continuada de Enfermagem (ECE) é um processo educativo ou de formação complementar, direcionado aos profissionais que trabalham nas diversas áreas. Ele é entendido como um processo ativo e permanente, que envolve metodologias ativas de ensino-aprendizagem, destinado a atualizar a capacitação dos profissionais ou grupos, frente à evolução das novas tecnologias, com uma nova visão humanista, direcionado as necessidades sociais e institucionais (FLAVIA F, et al., 2017).

Diante do exposto, todos os pontos citados e associados a assistência de enfermagem humanizada, fundamentam o presente artigo, que objetiva através de uma revisão de literatura, reunir conhecimentos e pesquisas, para que seja possível entender e compreender fatores que estejam envolvidos na promoção e integralidade da assistência de enfermagem prestada ao paciente em uma UTI, atentando para a percepção da equipe de enfermagem sobre a importância da assistência humanizada e os possíveis fatores que interferem nesse processo.

\section{MÉTODOS}

Trata-se de uma pesquisa descritiva, com abordagem qualitativa, utilizando como técnica a revisão integrativa de literatura (RIL) uma vez que ela possibilita descobrir se já foi produzido estudos direcionados ao mesmo tema, em anos distintos, impulsionando o aprendizado e a escrita cientifica (SOARES CB, et al., 2014). Tendo como base a seguinte pergunta norteadora: Quais as estratégias utilizadas para uma assistência de enfermagem humanizada em unidades de terapia intensiva?

A revisão integrativa de literatura pode resumir o passado, seja ele empírico ou teórico, tornando possível a compreensão mais ampla de determinada situação, fenômeno ou evento, além de possibilitar a inclusão de diferentes tipos de estudos, com diferentes abordagens metodológicas, conceitos, objetivos, fontes e ideais. 
Esse método torna possível utilizar a literatura cientifica e analítica com diversas metodologias (quantitativo e qualitativo), utilizando estudos sistematicamente revisados (SOARES CB, et al., 2014).

A pesquisa foi realizada em cinco momentos: identificação da problemática; estabelecimento dos critérios de elegibilidade e inelegibilidade dos artigos; avaliação dos artigos; interpretação e análise dos resultados obtidos após a seleção; e por último a síntese completa dos dados selecionados para revisão. O método utilizado para seleção dos artigos foi a leitura do título e do resumo; quando necessário, a leitura completa dos artigos.

A pesquisa foi realizada entre os meses de dezembro de 2020 e fevereiro de 2021, nas bases de dados da Biblioteca Virtual de Saúde (BVS) e Scientific Electronic Library Online (SCIELO), utilizando os seguintes descritores em saúde: Enfermagem AND Humanização AND Unidade de Terapia Intensiva. As bases de dados foram selecionadas por possuírem uma maior acessibilidade de dados publicados em diferentes revistas. Foram incluídos na pesquisa somente artigos que possuíam permissão do comitê de ética, quando se tratava de estudos realizadas com seres humanos. Foram descartados resumos e artigos que apresentavam incongruências quando não estavam relacionados ao tema desejado.

Os critérios de elegibilidade foram: publicações disponíveis na íntegra do banco de dados de forma gratuita, nos idiomas português e inglês, publicados dentro do período de 2015 a 2020, que estivessem relacionados ao tema abordado. Os critérios de inelegibilidade foram: as publicações que estavam indisponíveis na íntegra de gratuita, fora dos idiomas português e inglês, publicados nos anos anteriores a 2015 e que não estavam relacionados ao tema desejado.

\section{RESULTADOS}

Utilizando os descritores em saúde "Enfermagem AND Humanização AND Unidade de Terapia Intensiva" foram encontradas 280 publicações na totalidade, sendo 45 no banco de dados da SCIELO e 235 no BVS. Ao adicionar os critérios de artigos publicados em português e inglês esse número reduziu para 254, após o filtro de publicações entre os anos 2015 a 2020, o número reduziu para 74. Após a leitura, análise e interpretação dos artigos, 15 publicações destinadas ao objetivo desejado foram selecionadas para compor essa pesquisa (Figura 1).

Em relação aos tipos de estudos selecionados para compor esta revisão, nove são do tipo descritivo $(59,94 \%)$, cinco transversais $(33,3 \%)$ e um documental $(6,66 \%)$, sendo que em todos os artigos foram utilizadas a abordagem do tipo qualitativa (Gráfico 1).

Gráfico 1 - Tipos de estudo encontrados

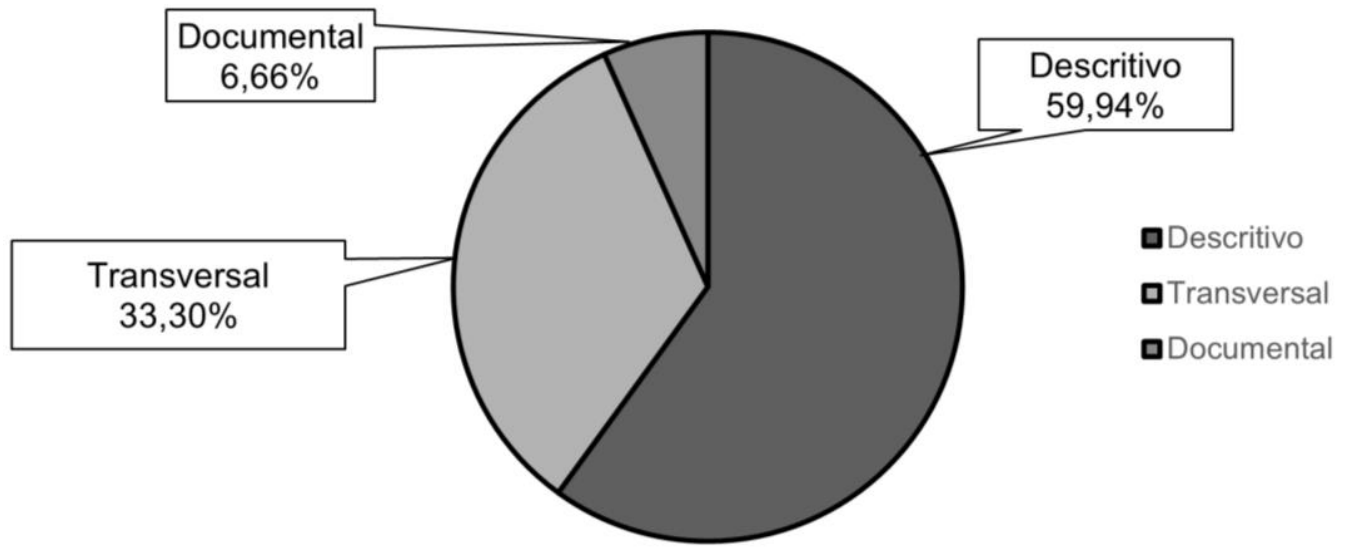

Fonte: Barbosa IEB, et al., 2021.

No presente estudo não houve periódico ou revista dominante, mas como maiores contribuições, tivemos a Revista Brasileira de Enfermagem (2016, 2017 e 2018) e a Cuidado é Fundamental Online (2015, 2016 e 
2018), ambas com 3 publicações cada. Outras revistas foram a Revista Mineira de Enfermagem (2016 e 2018), Cogitare Enfermagem (2015), Estudos de Psicologia I Campinas (2015), Revista da Escola de Enfermagem da Universidade de São Paulo (2016), Revista Baiana de Enfermagem (2018), CuidArte Enfermagem (2019), Revista Brasileira Promoção da Saúde (2019) e Revista de Enfermagem da Universidade Federal de Pernambuco (2019). No ano de 2020 não foram encontradas publicações relacionadas ao objetivo do estudo, bem como à pergunta norteadora.

Figura 1 - Fluxograma das etapas da seleção dos artigos para revisão.

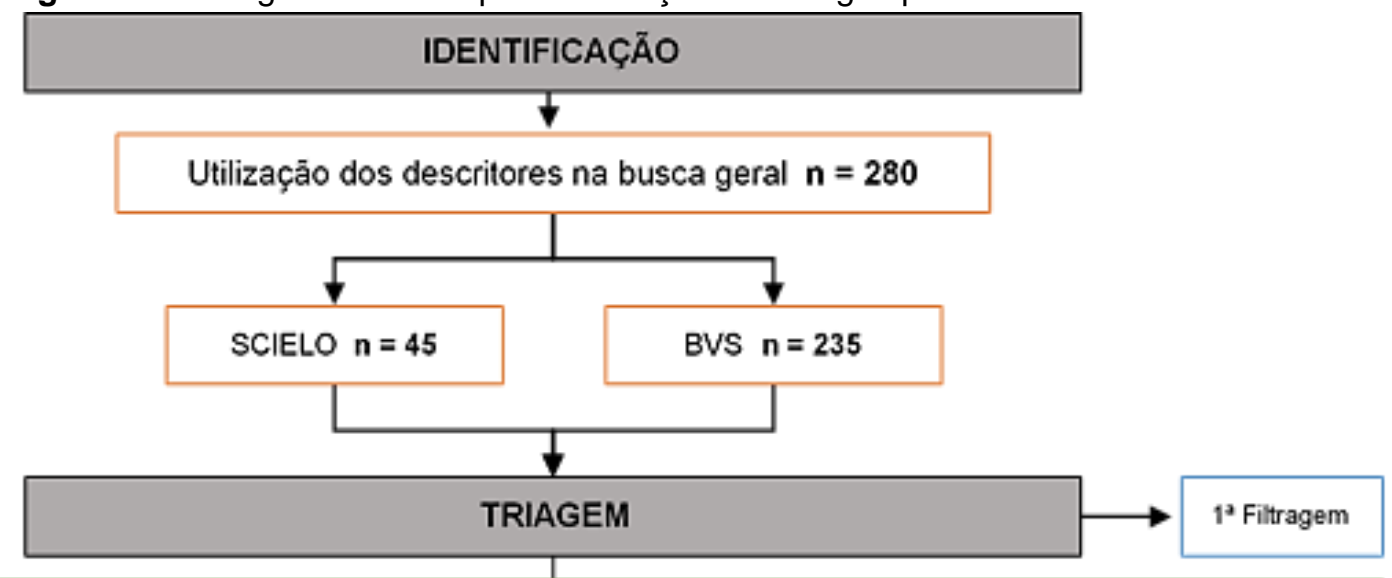

$\rightarrow$ Aplicaçăo dos critérios de inclusăo exclusão:

$\rightarrow$ Exclusăo de duplicaçŏes entre as bases de dados;

$\rightarrow$ Exclusăo de artigos publicados em outros idiomas que năo sejam inglês e português:

$\rightarrow$ Exclusão de estudos secundários.

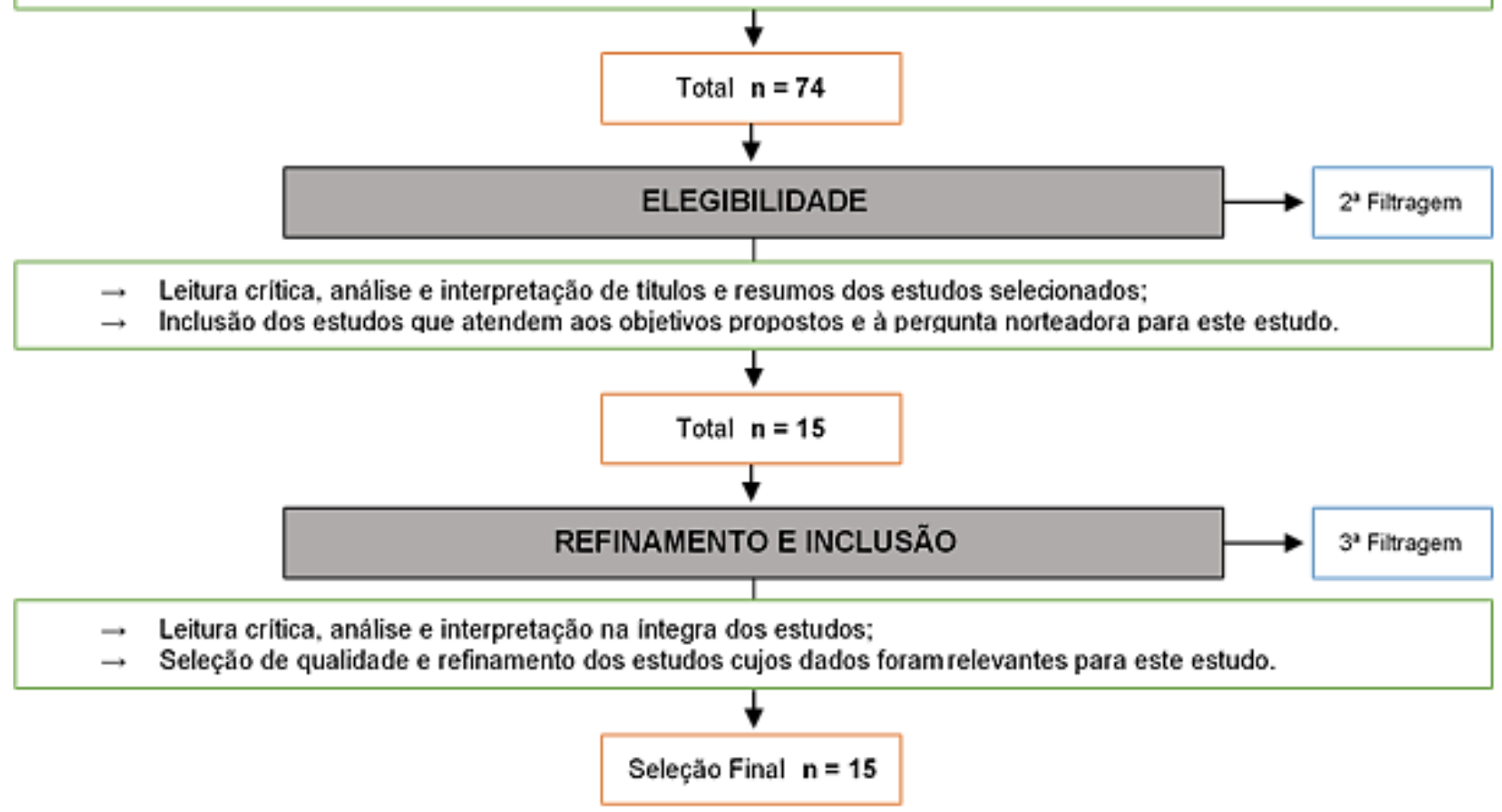

Fonte: Barbosa IEB, et al., 2021.

Para melhor organização e planejamento dos estudos escolhidos para esta revisão, foram levantadas informações como autor, ano, título, objetivo, tipo de estudo e publicação, facilitando o processo de interpretação dos resultados obtidos e dos artigos selecionados para a composição do estudo em síntese (Quadro 1). Seguidamente, os artigos foram categorizados e agrupados em tópicos para melhor discussão e compreensão. 
Quadro 1 - Artigos selecionados para revisão.

\begin{tabular}{|c|c|c|c|c|c|}
\hline $\mathbf{N}^{\circ}$ & Autor/Ano & Título & Objetivo & Estudo & Publicação \\
\hline 1 & $\begin{array}{l}\text { Flavia F, et al. } \\
\qquad(2017)\end{array}$ & $\begin{array}{l}\text { Humanização na Terapia Intensiva: } \\
\text { percepção do familiar e do } \\
\text { profissional de saúde }\end{array}$ & $\begin{array}{c}\text { Compreender as percepções de familiares e } \\
\text { profissionais de saúde sobre humanização na } \\
\text { Unidade Terapia Intensiva (UTI) para direcionar a } \\
\text { uma ação educativa. }\end{array}$ & $\begin{array}{l}\text { Exploratório- } \\
\text { descritivo } \\
\text { qualitativo }\end{array}$ & $\begin{array}{l}\text { Revista Brasileira de } \\
\text { Enfermagem (REBEn) }\end{array}$ \\
\hline 2 & $\begin{array}{l}\text { Medeiros AC, et } \\
\text { al. (2016) }\end{array}$ & $\begin{array}{l}\text { Integralidade e humanização na } \\
\text { gestão do cuidado de enfermagem } \\
\text { na Unidade de Terapia Intensiva }\end{array}$ & $\begin{array}{l}\text { Identificar os elementos capazes de promover a } \\
\text { integralidade e humanização na gestão do } \\
\text { cuidado de enfermagem na Unidade de Terapia } \\
\text { Intensiva, com enfoque ecossistêmico }\end{array}$ & $\begin{array}{l}\text { Documental } \\
\text { Qualitativo }\end{array}$ & $\begin{array}{l}\text { Revista da Escola de } \\
\text { Enfermagem (USP) }\end{array}$ \\
\hline 3 & $\begin{array}{l}\text { Evangelista VC, } \\
\text { et al. (2016) }\end{array}$ & $\begin{array}{l}\text { Equipe multiprofissional de terapia } \\
\text { intensiva: humanização e } \\
\text { fragmentação do processo de } \\
\text { trabalho }\end{array}$ & $\begin{array}{c}\text { Compreender o significado do cuidado } \\
\text { humanizado em unidades de terapia intensiva } \\
\text { considerando a vivência da equipe } \\
\text { multiprofissional. }\end{array}$ & $\begin{array}{l}\text { Descritiva e } \\
\text { exploratória de } \\
\text { caráter } \\
\text { qualitativo. }\end{array}$ & $\begin{array}{l}\text { Revista Brasileira de } \\
\text { Enfermagem (REBEn) }\end{array}$ \\
\hline 4 & $\begin{array}{l}\text { Cláudia PR, et } \\
\text { al. (2015) }\end{array}$ & $\begin{array}{l}\text { Concepções de humanização de } \\
\text { profissionais em Unidades de } \\
\text { Terapia Intensiva Neonatal }\end{array}$ & $\begin{array}{c}\text { Investigar a concepção de humanização e de } \\
\text { cuidado humanizado da equipe de profissionais } \\
\text { da UTIN, bem como o relato de suas práticas de } \\
\text { assistência ao RN. }\end{array}$ & $\begin{array}{l}\text { Transversal } \\
\text { Qualitativo }\end{array}$ & $\begin{array}{c}\text { Estudos de Psicologia I } \\
\text { Campinas }\end{array}$ \\
\hline 5 & $\begin{array}{l}\text { Carlos CZSF, et } \\
\quad \text { al. (2019) }\end{array}$ & $\begin{array}{l}\text { Estratégias do enfermeiro } \\
\text { intensivista neonatal frente à } \\
\text { Humanização do cuidado }\end{array}$ & $\begin{array}{c}\text { Descrever as estratégias utilizadas pelo } \\
\text { enfermeiro intensivista neonatal no processo de } \\
\text { humanização do cuidado }\end{array}$ & Descritivo & CuidArte Enfermagem \\
\hline 6 & $\begin{array}{l}\text { Silveira RE, et } \\
\text { al. (2015) }\end{array}$ & $\begin{array}{l}\text { Educação em saúde e prática } \\
\text { humanizada da enfermagem em } \\
\text { unidades de terapia intensiva: } \\
\text { estudo bibliométrico }\end{array}$ & $\begin{array}{l}\text { Abordar a participação do Enfermeiro enquanto } \\
\text { principal cuidador e educador em saúde inserido } \\
\text { na UTI. }\end{array}$ & Descritivo & $\begin{array}{l}\text { Cuidado é Fundamental } \\
\text { Online }\end{array}$ \\
\hline 7 & $\begin{array}{l}\text { Santos EL, et al. } \\
\qquad(2018)\end{array}$ & $\begin{array}{l}\text { Assistência humanizada: } \\
\text { percepção do enfermeiro } \\
\text { intensivista }\end{array}$ & $\begin{array}{c}\text { Analisar a percepção do enfermeiro intensiva } \\
\text { sobre a assistência humanizada. }\end{array}$ & $\begin{array}{l}\text { Transversal } \\
\text { Qualitativo }\end{array}$ & $\begin{array}{l}\text { Revista Baiana de } \\
\text { Enfermagem }\end{array}$ \\
\hline
\end{tabular}




\begin{tabular}{|c|c|c|c|c|c|}
\hline $\mathbf{N}^{\circ}$ & Autor/Ano & Título & Objetivo & Estudo & Publicação \\
\hline 8 & $\begin{array}{l}\text { Reis CCA, et al. } \\
\qquad(2016)\end{array}$ & $\begin{array}{l}\text { Humanização do cuidado nas } \\
\text { unidades de terapia intensiva: } \\
\text { revisão integrativa }\end{array}$ & $\begin{array}{l}\text { Identificar o que a literatura científica nacional tem } \\
\text { abordado acerca da humanização do cuidado nas } \\
\text { Unidades de Terapia Intensiva em bases de } \\
\text { dados online, no período de } 2009 \text { a } 2013 \text {. }\end{array}$ & $\begin{array}{l}\text { Descritivo } \\
\text { Qualitativo }\end{array}$ & $\begin{array}{c}\text { Cuidado é Fundamental } \\
\text { Online }\end{array}$ \\
\hline 9 & $\begin{array}{l}\text { Carli BS, et al. } \\
\qquad(2018)\end{array}$ & $\begin{array}{l}\text { O tema da humanização na terapia } \\
\text { intensiva em pesquisas na saúde }\end{array}$ & $\begin{array}{l}\text { Sistematizar estudos indexados no SCIELO e } \\
\text { Medline, sobre humanização em Unidade de } \\
\text { Terapia Intensiva. }\end{array}$ & $\begin{array}{l}\text { Descritivo } \\
\text { Qualitativo }\end{array}$ & $\begin{array}{l}\text { Cuidado é Fundamental } \\
\text { Online }\end{array}$ \\
\hline 10 & $\begin{array}{l}\text { Castro AS, et al. } \\
\qquad(2019)\end{array}$ & $\begin{array}{c}\text { Percepções da equipe de } \\
\text { enfermagem acerca da } \\
\text { humanização em Terapia intensiva }\end{array}$ & $\begin{array}{c}\text { Conhecer as percepções da equipe de } \\
\text { Enfermagem acerca da humanização da } \\
\text { assistência em Unidade de Terapia Intensiva. }\end{array}$ & $\begin{array}{l}\text { Transversal } \\
\text { Qualitativo }\end{array}$ & $\begin{array}{l}\text { Revista Brasileira } \\
\text { Promoção da Saúde }\end{array}$ \\
\hline 11 & $\begin{array}{l}\text { Martins TJ, et al. } \\
\quad(2015)\end{array}$ & $\begin{array}{c}\text { Humanização no processo de } \\
\text { trabalho na Percepção de } \\
\text { enfermeiros de unidade de terapia } \\
\text { Intensiva }\end{array}$ & $\begin{array}{c}\text { Identificar os fatores que propiciam e dificultam a } \\
\text { humanização entre os trabalhadores de } \\
\text { enfermagem, na percepção de enfermeiros de } \\
\text { uma unidade de terapia intensiva }\end{array}$ & $\begin{array}{l}\text { Descritivo } \\
\text { Qualitativo }\end{array}$ & Cogitare Enfermagem \\
\hline 12 & $\begin{array}{l}\text { Costa JVS, et } \\
\text { al. (2019) }\end{array}$ & $\begin{array}{c}\text { Humanização da assistência } \\
\text { neonatal na ótica dos profissionais } \\
\text { da Enfermagem }\end{array}$ & $\begin{array}{l}\text { Identificar a percepção da equipe de Enfermagem } \\
\text { sobre a humanização da assistência prestada em } \\
\text { uma Unidade de Terapia Intensiva Neonatal. }\end{array}$ & $\begin{array}{l}\text { Descritivo } \\
\text { Qualitativo }\end{array}$ & $\begin{array}{l}\text { Revista de Enfermagem } \\
\text { UFPE online. }\end{array}$ \\
\hline 13 & $\begin{array}{l}\text { Rodrigues AC, } \\
\text { et al. (2016) }\end{array}$ & $\begin{array}{c}\text { Humanização da assistência na } \\
\text { unidade de terapia intensiva } \\
\text { Pediátrica: perspectiva da equipe } \\
\text { de enfermagem }\end{array}$ & $\begin{array}{c}\text { Analisar a visão da equipe de enfermagem sobre } \\
\text { a humanização da assistência às crianças e } \\
\text { famílias na Unidade de Terapia Intensiva } \\
\text { Pediátrica (UTIP) }\end{array}$ & $\begin{array}{l}\text { Transversal } \\
\text { Qualitativa }\end{array}$ & $\begin{array}{l}\text { Revista Mineira de } \\
\text { Enfermagem (REME) }\end{array}$ \\
\hline 14 & $\begin{array}{l}\text { Noda LM, et al. } \\
\qquad(2018)\end{array}$ & $\begin{array}{l}\text { A humanização em unidade de } \\
\text { terapia intensiva neonatal sob a } \\
\text { Ótica dos pais }\end{array}$ & $\begin{array}{c}\text { Compreender os significados de humanização da } \\
\text { assistência sob a ótica de pais de recém-nascidos } \\
\text { internados em uma Unidade de Terapia Intensiva } \\
\text { Neonatal. }\end{array}$ & $\begin{array}{l}\text { Descritivo e } \\
\text { exploratório, de } \\
\text { natureza } \\
\text { qualitativa }\end{array}$ & $\begin{array}{l}\text { Revista Mineira de } \\
\text { Enfermagem (REME) }\end{array}$ \\
\hline 15 & $\begin{array}{l}\text { Michelan VCA, } \\
\text { et al. (2018) }\end{array}$ & $\begin{array}{l}\text { Percepção da humanização dos } \\
\text { trabalhadores de enfermagem em } \\
\text { terapia intensiva }\end{array}$ & $\begin{array}{c}\text { Compreender a percepção dos trabalhadores de } \\
\text { enfermagem que atuam em Unidade de Terapia } \\
\text { Intensiva (UTI) a respeito da humanização no } \\
\text { ambiente de trabalho. }\end{array}$ & $\begin{array}{l}\text { Transversal } \\
\text { Qualitativa }\end{array}$ & $\begin{array}{l}\text { Revista Brasileira de } \\
\text { Enfermagem (REBEn) }\end{array}$ \\
\hline
\end{tabular}

Fonte: Barbosa IEB, et al., 2021. 


\section{DISCUSSÃO}

Para que seja possível uma compreensão mais profunda sobre questões que envolvam atendimentos assistenciais humanizados em UTI, tona-se necessária uma abordagem mais ampla sobre os múltiplos aspectos e dimensões que estejam envolvidas ao ser humano. Diante disso, é extremamente válido entender primeiramente os referenciais que envolvem esse processo, para que assim seja desenvolvida uma visão mais ampliada das bases e elementos que compõem esse ambiente, facilitando assim uma visão holística sobre a problemática (CARLI BS, et al., 2018).

\section{Fatores envolvidos na promoção e integralidade da assistência humanizada na UTI}

Um atendimento humanizado na UTI não se restringe somente às técnicas que envolvam como fazer, mas sim às ações que expressam uma atitude de cuidados preexistentes envolvendo o paciente e seus familiares. Ações que envolvam sensibilidade e prazer nas tarefas executadas, são fatores primordiais para realização de um atendimento humanizado, facilitando a interação com o paciente, disponibilizando assim a introdução de diálogos mais próximos a ambos (NODA LM, et al., 2018).

A temática referente à humanização é um processo que exige uma compreensão que vai além das ações assistenciais prestadas ao paciente, ou seja, abrange de modo geral os sistemas e o ambiente onde a mesma se encontra. Diante disso, cabe a cada profissional de enfermagem se empenhar em prestar uma assistência humana, atentando sempre para as questões que podem fragilizar e interferir nessa ação, colocando-se sempre no lugar da pessoa enferma, tratando sempre com respeito, vendo o paciente como pessoa humana, não somente como um serviço ou um procedimento que precisa ser realizado (Martins TJ, et al., 2015).

A assistência de enfermagem humanizada é direcionada principalmente para os princípios éticos, envolvendo a singularidade de cada profissional, visando ações e estratégias que viabilizem o cuidado em todas suas dimensões, levando a recuperação do cliente e valorizando os sujeitos que estão inseridos no processo do cuidar. Nesse contexto, torna-se visível que a assistência humanizada é um processo contínuo, que passa por diversas adaptações, podendo ser recorrentes de curto ou longo prazo, sofrendo variação de entre os profissionais (REIS CCA, et al., 2016).

\section{Concepção dos profissionais sobre a assistência de enfermagem humanizada}

Segundo Cláudia PR, et al. (2015), os profissionais definem a humanização como o oposto do modelo de assistência médico-tecnicista, em que fatores como o resgate de aspectos emocionais e fortalecimento das relações interpessoais são características integrais e importantes nesse processo, priorizando a cultura assistencial na produção de mudanças envolvendo a assistência humanizada. Os profissionais entendem o processo de humanização como uma boa relação interpessoal entre o paciente e o profissional, resultada de uma assistência respeitosa prestada, acolhimento e sobretudo uma comunicação efetiva.

Na pesquisa de Rodrigues CA, et al. (2016) realizada dentro de uma UTI pediátrica, relataram que os profissionais realizam apenas uma parte das ações assistenciais de maneira humanizada e que essa temática está voltada principalmente para o acolhimento das crianças e dos pais, assim como também no esclarecimento de dúvidas com os responsáveis, realizando acompanhamento e visitas quando necessário. Outras ações também foram listadas, como: dispor de conforto para o paciente e seus familiares.

Segundo Michelan VCA, et al. (2018), a assistência de enfermagem envolve três pilares primordiais nesse processo, sendo: a condição de trabalho, gestão de pessoas e o processo gerencial na UTI. Nessa temática foi observado que o processo assistencial está diretamente envolvido também no acolhimento e trabalho em equipe dos profissionais, relatando que humanizar não é só a forma de trabalhar, mas sim assistir o paciente como um ser biopsicossocial.

Entretanto, dentro da área da UTI neonatal, Costa JVS, et al. (2019) abordaram que, segundo os profissionais, o cuidado humanizado é o papel central na vida e recuperação dos pacientes, focando nos cuidados e na segurança dos procedimentos realizados. Acrescentou-se também o cuidar em todas suas dimensões da prática social, na tentativa de minimizar procedimentos errôneos, ressaltando assim 0 atendimento humanizado essencial para o bebê e seus familiares. 
Segundo Castro AS, et al. (2019), os profissionais compreendem que a assistência humanizada é primordial para melhora do quadro clínico do paciente, e que a comunicação é uma grande aliada nesse processo, sendo necessário se comunicar de maneira clara e concisa com pacientes e seus familiares, assim como também com a equipe multiprofissional que atuam nessa área hospitalar.

Corroborando com o presente estudo, Castro AS, et al. (2019) concluíram que uma comunicação efetiva é essencial para o processo de humanização, pois tanto pacientes, familiares e profissionais possuem um déficit nesse quesito. Comunicar-se de maneira clara e concisa aumentam as chances de uma qualidade assistencial satisfatória. Contudo, esse cuidado não demanda somente práticas assistenciais e de orientação, mas sim tratar o paciente integralmente, com respeito, dedicação e afetividade.

Diante disso, observa-se que os profissionais possuem bastante conhecimentos sobre a assistência de enfermagem humanizada, assim como também sobre a sua relevância para melhoria do atendimento ao paciente, porém não as colocam em prática da maneira correta. Essa não adesão pode estar relacionada aos fatores pessoais, profissionais e institucionais.

\section{Fatores que contribuem para assistência de enfermagem humanizada}

Segundo Evangelista VC, et al. (2016), a comunicação é um processo importante nessa ação, sendo válida no esclarecimento de dúvidas entre familiares e pacientes, tais como saber quais as chances de sobrevida, ter suas perguntas respondidas de forma clara, quem são os profissionais que estão envolvidos na recuperação e principalmente receber informações que possam ser compreendias de maneira clara. Apresentou também a relevância do trabalho em equipe dos profissionais de enfermagem, constatando um fator de interação e organização no setor de trabalho na UTI, tornando possível uma maior articulação de ações humanizadas entre todos os profissionais e os pacientes.

Na pesquisa de Rodrigues CA, et al. (2016), destacaram-se fatores que envolvem o respeito, escuta, conforto e presença da família. Os profissionais relataram sobre algumas condições que favorecem para esse processo, como: o setor bem relacionado com toda equipe, bem-estar profissional, valorização do trabalho e, principalmente, a capacitação e formação correta dos profissionais, respeitando o paciente em todas suas dimensões.

Segundo Costa JVS, et al. (2019), os principais fatores que podem contribuir no processo de aprimoramento da assistência de enfermagem humanizada são: a gestão ser presente nos serviços assistências, experiência profissional, equipe capacitada e responsável, força de vontade dos profissionais, boa comunicação e relacionamento interpessoal com todos os profissionais, a disposição de materiais e instrumentos necessários para realização dos procedimentos, tendo como foco principal o afeto dos trabalhadores pela profissão escolhida.

Flavia F, et al. (2017), aborda em sua pesquisa sobre fatores importantes para realização da assistência de enfermagem humanizada na UTI pediátrica, dentre eles destacam-se: o respeito, conforto, observação, empatia, escuta, privacidade, presença da família e acompanhantes e principalmente a infraestrutura da instituição. Destacou-se também sobre a jornada de trabalho, que é algo importante nesse processo.

Segundo Michelan VCA, et al. (2018), alguns aspectos que podem influenciar positivamente na assistência de enfermagem humanizada são: garantir visitas diárias em mais de um turno, capacitar os profissionais periodicamente, aliar teorias aos procedimentos, ter recurso para realização de procedimentos seguros e de qualidade e atuar com sintonia e respeito mútuo com os profissionais, pacientes, familiares e acompanhantes.

Dentre os pontos citados, em cinco das pesquisas selecionadas para revisão, os principais fatores que contribuem para assistência de enfermagem humanizada estão relacionados a cinco pontos principais, sendo a comunicação interpessoal entre os profissionais a mais abordada nas pesquisas, seguido da infraestrutura da área hospitalar, capacitação correta dos profissionais, bem como o prazer pela atividade exercida e, por fim, as ações voltadas para o respeito com os pacientes e seus familiares.

\section{Fatores que dificultam a assistência de enfermagem humanizada}

Na pesquisa de Rodrigues CA, et al. (2016), fatores como o despeito do conhecimento, ausência da atualização científica, redução do número de funcionários e alta demanda de procedimentos em pouco tempo, 
são os principais preditores que interferem para que a assistência de enfermagem seja feita de forma humanizada, como também a idade e o tempo de serviço naquela unidade hospitalar, que podem interferir significativamente nesse processo.

Segundo Costa JVS, et al. (2019), a falta de comunicação entre os pacientes e os profissionais é um dos principais fatores que dificultam essa assistência. Destacou-se também a falta de uma boa relação interpessoal, instrumentos e materiais disponíveis, grande demanda de serviços de saúde, padronização dos medicamentos, falta de conscientização da população e principalmente a falta de capacitação dos profissionais, que é algo extremamente importante nesse processo.

Martins TJ, et al. (2015), abordam fatores que podem influenciar nesse processo, como: a redução do quadro de funcionários, tempo e alta demanda de pacientes, falta de informação teórico-prática, infraestrutura, limitação do autoconhecimento, presença de acompanhantes e o desinteresse profissional. Alguns dos fatores são correspondentes às outras pesquisas já citadas acima por outros autores.

Observou-se com maior prevalência nas pesquisas selecionadas que os principais fatores que dificultam a assistência de enfermagem humanizada estão relacionados à falta de conhecimento e à diminuição do quadro de funcionários, assim como a infraestrutura e a falta de um bom relacionamento interpessoal entre os profissionais, dificultando assim na assistência prestada.

\section{CONSIDERAÇÕES FINAIS}

A assistência de enfermagem humanizada contribui para a melhora do paciente dentro das UTIs, logo, cabe aos profissionais analisarem suas práticas e se capacitarem frente a essa situação, avaliando teorias e programas de educação continuada para garantir a segurança nos procedimentos prestados, priorizando um manejo e atendimento equânime e eficiente. Humanizar dentro de uma UTI está relacionado principalmente na melhora das práticas cuidadosas, com ética, diálogo e autonomia aos pacientes e seus familiares, pois simples atitudes podem contribuir significativamente nesse processo. Portanto, o interesse na capacitação dos profissionais, por meio da educação continuada pelas instituições de saúde públicas e privadas, que visem a melhora no atendimento frente às tecnologias, é relevante.

\section{REFERÊNCIAS}

1. BARBOSA IEB, et al. Segurança do paciente: principais eventos adversos na Unidade Terapia Intensiva. Revista Eletrônica Acervo Saúde, 2021; 13(2): e6454.

2. COSTA JVS, et al. Humanização da assistência neonatal na ótica dos profissionais de enfermagem. Rev enferm UFPE on line, 2019; 13: e242642

3. CARLI BS, et al. The humanization theme in intensive care in health studies. Rev Fund Care Online. 2018.

4. CORBANI NMS, et al. Humanização do cuidado de enfermagem: o que é isso? Rev Bras Enferm, 2009; 62(3): 34954.

5. EVANGELISTA VC, et al. Multidisciplinary team of intensive therapy: humanization and fragmentation of the work process. Rev Bras Enferm, 2016; 69(6): 1037-44.

6. LUIZ FF,et al. Humanização na terapia intensiva: percepção do familiar e do profissional de saúde. Rev Bras Enferm, 2017; 70(5): 1095-103.

7. LUIZ FF, et al. Humanization in the Intensive Care: perception of family and healthcare professionals. Rev Bras Enferm, 2017; 70(5): 1040-7.

8. MACHADO ER, et al. Humanização em UTI: sentidos e significados sob a ótica da equipe de saúde. Rev Enferm do Cent-Oeste Min, 2016; 6(3): 2342-8.

9. MASSAROLI R, et al. Trabalho de enfermagem em unidade de terapia intensiva e sua interface com a sistematização da assistência. Esc Anna Nery Rev Enferm, 2015; 19(2): 252-8.

10. MALTA DC, et al. Akerman M. Brazil's Unified Health System and the National Health Promotion Policy: prospects, results, progress and challenges in times of crisis. Ciênc Saúde Colet, 2018; 23(6): 1799-809.

11. MINISTÉRIO DA SAÚDE BR, Secretaria de Assistência à Saúde. Cartilha Humaniza SUS. Política Nacional de Humanização - PNH. Brasília: Ministério da Saúde, 2015.

12. MICHELAN VCA, et al. Perception of nursing workers humanization under intensive therapy. Rev Bras Enferm, 2018; 71(2): 372-8.

13. MEDEIROS AC, et al. Comprehensiveness and humanization of nursing care management in the Intensive Care Unit. Rev Esc Enferm USP, 2016; 50(5): 816-822. 
14. NODA LM, et al. A humanização em Unidade de Terapia Intensiva Neonatal sob a ótica dos pais. REME - Rev Min Enferm, 2018.

15. PASCUCI L, et al. Humanization in a hospital: a change process integrating individual, organizational and social dimensions. J Health Manag, 2017; 19(2): 1-20.

16. PEDUZZI M, et al. O auxiliar e o técnico de enfermagem: categorias profissionais diferentes e trabalhos equivalentes. Rev Bras Enferm, 2004; 57(4):425-9

17. PENNA CMM, et al. Acolhimento: triagem ou estratégia para universalidade do acesso na atenção à saúde? REME - Rev Min Enferm, 2014; 18(4):815-22.

18. RODRIGUES AC, et al., Humanização da assistência na unidade de terapia intensiva pediátrica: perspectiva da equipe de Enfermagem. REME - Rev Min Enferm, 2016.

19. ROSEIRO, CP e PAULA, KMP. Concepções de humanização de profissionais em Unidades de Terapia Intensiva Neonatal. Estud. psicol. (Campinas), 2015; 32(1): 109-119.

20. SANTOS ES, et al. Acolhimento e processo educativo em saúde a familiares de pacientes internados em UTI adulto. Ciênc Cuid Saúde, 2016; 15(4): 639-46.

21. SANTOS EL, et al. Assistência humanizada: percepção do enfermeiro intensivista. Rev baiana enferm, 2018; 32: e23680.

22. SILVA JC, et al. Rede de atenção à saúde: percepção de usuárias do Sistema Único de Saúde. Rev Bras Promoç Saúde, 2016; 29: 44-50.

23. SILVEIRA DA, et al. Health education and humanized practice of nursing at intensive care units: bibliometric study. Revista de Pesquisa: Cuidado é Fundamental Online, 2015. 7(1): 2113-2122. 